\title{
The Mechanism of cAMP-Mediated Enhancement at a Cerebellar Synapse
}

\author{
Chinfei Chen and Wade G. Regehr \\ Department of Neurobiology, Harvard Medical School, Boston, Massachusetts 02115
}

Increases in cAMP have been shown previously to enhance the strength of the granule cell to Purkinje cell synapse. We have examined the mechanisms underlying this enhancement in rat cerebellar brain slices. Elevation of cAMP levels by forskolin increased synaptic currents in a dose-dependent manner. Fluorometric calcium measurements revealed that forskolin did not affect presynaptic calcium influx or resting calcium levels. The waveform of the presynaptic volley was also unaltered, indicating that changes in the presynaptic action potential did not contribute to synaptic enhancement. However, forskolin enhanced the frequency but not the size of spontaneous miniature EPSCs. There was a one-to-one correspondence between increases of spontaneous and evoked neurotransmitter release.

Many neuromodulators can rapidly change the strength of granule cell to Purkinje cell synapses (Sabatini and Regehr, 1995; Dittman and Regehr, 1996; Salin et al., 1996). One such modulator is cAMP. Elevation of cAMP levels leads to a large and rapid presynaptic enhancement, presumably by activating protein kinase A (PKA) (Salin et al., 1996). At present, the mechanisms by which cAMP modulates this synapse are not known.

At other synapses, changes in cAMP levels act via a variety of mechanisms to modify synaptic strength. Spike broadening contributes to cAMP-dependent enhancement of the Aplysia sensorimotor synapses of the abdominal ganglion (Byrne and Kandel, 1996). Presynaptic mechanisms independent of calcium have been implicated in cAMP-dependent modulation in Aplysia, the crayfish neuromuscular junction, both inhibitory and excitatory hippocampal synapses, and inhibitory cerebellar synapses (Goy and Kravitz, 1989; Dale and Kandel, 1990; Delaney et al., 1991; Llano and Gerschenfeld, 1993; Chavez-Noriega and Stevens, 1994; Weisskopf et al., 1994; Capogna et al., 1995; Trudeau et al., 1996; Kondo and Marty, 1997). In the hippocampus, cAMP can also act postsynaptically by modifying ligand-gated receptors (Greengard et al., 1991). In addition, cAMP has been shown to modulate voltage-gated calcium channels (Hille, 1992). Thus, based on the diverse targets of cAMP, many possible mechanisms could be involved in cAMP-mediated enhancement at the granule cell to Purkinje cell synapse.

Understanding the actions of cAMP has important implica-

Received June 2, 1997; revised Aug. 6, 1997; accepted Aug. 29, 1997.

This work was supported by National Institutes of Health Grant R01-NS32405-01 and a grant from the Edward R. and Anne G. Lefler Center to W.R. and by the Howard Hughes Physician Postdoctoral Fellowship to C.C. We thank Pradeep Atluri, Bernardo Sabatini, Jeremy Dittman, and Bruce Peters for comments on this manuscript.

Correspondence should be addressed to Dr. Wade Regehr, Department of Neurobiology, Harvard Medical School, 220 Longwood Avenue, Boston MA 02115.

Copyright (C) 1997 Society for Neuroscience $0270-6474 / 97 / 178687-08 \$ 05.00 / 0$
These results suggest that forskolin increases release at this synapse via presynaptic mechanisms that do not alter calcium influx. The effect of forskolin on paired-pulse facilitation was examined to assess the relative contributions of changes in the probability of release $(p)$ and changes in the number of functional release sites $(n)$ to this form of enhancement. These experiments suggest that although small changes in $n$ cannot be excluded, most of the enhancement arises from increases in $p$.

Key words: synaptic modulation; cAMP; magnesium green; fura-2; mEPSC; forskolin; Purkinje cell; granule cell; pairedpulse facilitation

tions for a presynaptic form of long-term potentiation (LTP). It has been shown that presynaptic activity leads to a long-term enhancement of neurotransmitter release at the granule cell to Purkinje cell synapse (Salin et al., 1996). This presynaptic form of LTP involves cAMP and is similar to forms of use-dependent enhancement at the crayfish neuromuscular junction and mossy fiber synapses in the hippocampus (Dixon and Atwood, 1989; Huang et al., 1994; Weisskopf et al., 1994; Salin et al., 1996). At these synapses, changes in the probability of release $(p)$ and in the number of release sites $(n)$ have both been shown to contribute to LTP (Wojtowicz et al., 1988, 1994; Weisskopf et al., 1994; Weisskopf and Nicoll, 1995; Tong et al., 1996). However, at the granule cell to Purkinje cell synapse, the contributions of changes in $n$ and $p$ to cAMP-dependent modulation are not known.

Here we study the mechanisms of cAMP-dependent enhancement of transmission in rat cerebellar slices. Forskolin was used to elevate cAMP levels by activating adenylate cyclase (Zhang et al., 1997). We studied the effects of cAMP on fiber excitability, action potential waveform, presynaptic calcium influx, and vesicular release. We show that changes in presynaptic calcium influx are not involved in cAMP-mediated increases in synaptic strength. Moreover, we find that cAMP acts downstream from calcium influx to increase the probability of vesicular release.

\section{MATERIALS AND METHODS}

Electrophysiology. Transverse slices, $300 \mu \mathrm{m}$ thick, were cut from the cerebellar vermis of 9- to 15-d-old Sprague Dawley rats as described previously (Llano et al., 1991; Dittman and Regehr, 1996). Slices were maintained in a chamber with an external solution containing (in $\mathrm{mM}$ ): $125 \mathrm{NaCl}, 2.5 \mathrm{KCl}, 2 \mathrm{CaCl}_{2}, 1 \mathrm{MgCl}_{2}, 2.6 \mathrm{NaHCO}_{3}, 1.25 \mathrm{NaH}_{2} \mathrm{PO}_{4}$, and 25 glucose that was continuously bubbled with $95 \% \mathrm{O}_{2} / 5 \% \mathrm{CO}_{2}$. The external recording solution was the same with bicuculline added at $20 \mu \mathrm{M}$ to suppress inhibitory currents. Slices were continuously perfused with bubbled $95 \% \mathrm{O}_{2} / 5 \% \mathrm{CO}_{2}$ external solution at $1-3 \mathrm{ml} / \mathrm{min}$ in the recording chamber.

Synaptic EPSCs. Whole-cell recordings of Purkinje cells were obtained 
as described previously (Llano et al., 1991; Mintz et al., 1995), using $1.0-1.9 \mathrm{M} \Omega$ glass pipettes. The internal solution used in measurements of evoked (EPSC) and spontaneous miniature EPSCs (mEPSCs) contained (in mM): $110 \mathrm{Cs}_{2} \mathrm{SO}_{4}, 10$ EGTA, $4 \mathrm{CaCl}_{2}, 1.5 \mathrm{MgCl}_{2}, 5.5 \mathrm{MgSO}_{4}, 4$ $\mathrm{Na}_{2}$-ATP, 0.1 D600, and 10 HEPES, pH 7.4 (CsOH). After a high resistance seal was obtained, suction was applied lightly through the pipette to break through the membrane. The cell was then maintained at $-70 \mathrm{mV}$ for several minutes to allow diffusion of the internal solution into the cell body and dendrites. Access resistance $(<5 \mathrm{M} \Omega$ after series resistance compensation) and leak current were monitored continuously.

Parallel fibers were stimulated extracellularly by injecting a current pulse through a saline-filled glass electrode situated $0.5-1 \mathrm{~mm}$ from the recording electrode in the molecular layer. Synaptic currents were recorded at $20 \mathrm{kHz}$ and filtered at $1 \mathrm{kHz}$. Interstimulus intervals ranged from 15 to $30 \mathrm{sec}$ for experiments measuring evoked EPSCs. Experiments involving paired-pulse facilitation and mEPSCs had an interstimulus interval of $30 \mathrm{sec}$. Stimulus intensities ranged from 2.5 to $10 \mu \mathrm{A}$ and were $0.2-0.4 \mathrm{msec}$ in duration. To assess whether changes in fiber stimulus threshold could contribute to the enhancement seen in the presence of forskolin, we performed similar experiments in the presence of $0.5-1 \mathrm{~mm}$ kynurenic acid (KYN). In the presence of this low affinity competitive AMPA receptor antagonist, stimulus intensities ranging from 7 to $20 \mu \mathrm{A}$ were used to evoke EPSCs. The forskolin-mediated enhancement was unchanged with higher stimulus intensities $(n=6)$. Because there was no change in the forskolin effect in KYN, these experiments were included in the average time courses (see Fig. $1 B$ ).

Stock solutions of forskolin and 1,9-dideoxy-forskolin (Research Biochemicals, Natick, MA) were dissolved in dimethylsulfoxide (DMSO) and stored at $-80^{\circ} \mathrm{C}$ at $50-100 \mathrm{~mm}$ concentrations. A few minutes before the application of the drug, the stock solution was thawed and diluted directly into the bath reservoir. Control solutions always contained $0.1 \%$ DMSO. Kynurenic acid (Research Biochemicals) was solubilized in distilled water and stored at $-20^{\circ} \mathrm{C}$ at $100 \mathrm{~mm}$ stock concentrations. Dilution to final concentrations were made just before the start of the experiment. Recordings were performed between 22 and $25^{\circ} \mathrm{C}$.

Measurement of mEPSCs. mEPSCs were recorded in the whole-cell configuration using 1.3-1.7 $\mathrm{M} \Omega$ pipettes filled with the same internal solution used to measure evoked EPSCs. TTX $(0.25 \mu \mathrm{M})$ and $20 \mu \mathrm{M}$ bicuculline were included in the standard external solution to block action potential-evoked synaptic currents and IPSCs, respectively. In some experiments $50 \mu \mathrm{M} \mathrm{Cd}^{2+}$ was also included in the external solution to block voltage-gated calcium channels (Mintz et al., 1995). $\mathrm{Cd}^{2+} \mathrm{did}$ not reliably alter the basal mEPSC frequency or the effects of forskolin on mEPSC frequency; $50 \mu \mathrm{M}$ forskolin increased mEPSC frequency to $260 \pm 9 \%(n=3)$ in $\mathrm{Cd}^{2+}$ plus TTX compared with $280 \pm 8 \%(n=4)$ in the presence of TTX alone. $\mathrm{Cd}^{2+}$ was not routinely included in our experiments because it adversely affected the stability of recordings. Data were recorded in $20 \mathrm{sec}$ epochs, sampled at $2.5 \mathrm{kHz}$, and filtered at 500 $\mathrm{Hz}$ with an eight-pole Bessel filter (Frequency Devices, Haverhill, MA). Membrane potential was maintained at $-70 \mathrm{mV}$ unless otherwise indicated. To minimize the noise, we did not use series resistance compensation. Leak currents were -10 to $-100 \mathrm{pA}$. Events were counted and analyzed off-line using IGOR PRO software (Wavemetrics, Lake Oswego, OR) and custom macros (provided by Bernardo Sabatini). Inclusion criteria were a 4-6 pA amplitude threshold, a minimum rate of rise of $0.4 \mathrm{pA} / \mathrm{msec}$, and a decay time constant between 3 and $12 \mathrm{msec}$. Amplitude histograms were binned in $2 \mathrm{pA}$ intervals. Cumulative amplitude histograms were compared using the Kolmogorov-Smirnov test for significance. Statistical significance was assumed for $P \leq 0.05$.

Extracellular field recordings. Presynaptic volleys and calcium indicator fluorescence were recorded simultaneously. A saline-filled electrode (2$2.5 \mathrm{M} \Omega$ ) was placed near the surface of the parallel fibers within the $150-\mu \mathrm{m}$-diameter spot used to measure fluorescence and $400-700 \mu \mathrm{m}$ away from the stimulus site. The position of the electrode was chosen to maximize the amplitude of the presynaptic volley and the $\Delta F / F$ signal while maintaining separation between the volley and the stimulus artifact. Stimulus strength ranged from 40 to $120 \mu \mathrm{A}$.

Calcium-sensitive fluorescence measurements. Parallel fibers were labeled by local application of either magnesium green (Zhao et al., 1996) or fura-2 (Grynkiewicz et al., 1985) as described previously (Regehr and Tank, 1991; Regehr and Atluri, 1995). The fluorescence output was measured with a photodiode, sampled at $10 \mathrm{kHz}$, and filtered at $500 \mathrm{~Hz}$. The interstimulus interval was $30 \mathrm{sec}$. The $\Delta F / F$ ratio from a single stimulus was calculated and used as a linear measure of presynaptic calcium influx (Mintz et al., 1995; Regehr and Atluri, 1995). Resting
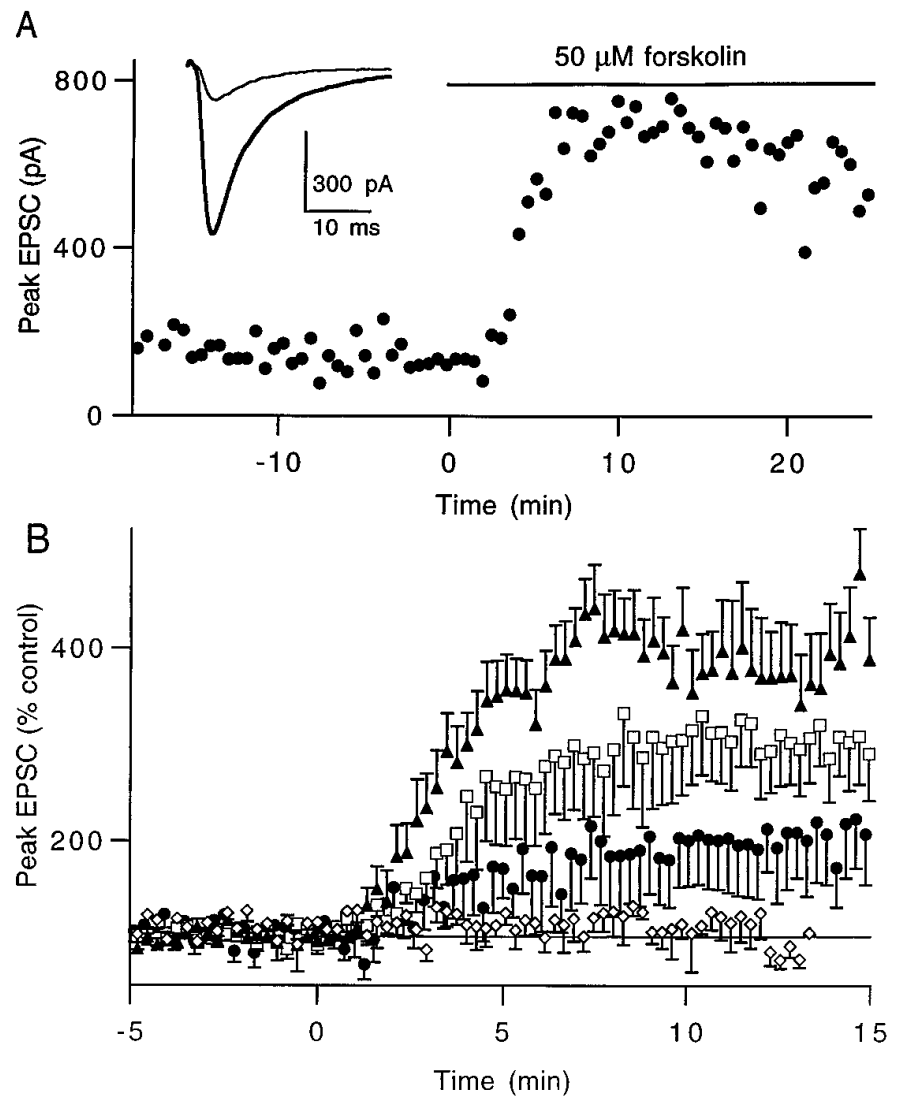

Figure 1. Forskolin enhances synaptic currents in a dose-dependent manner. $A$, Continuous application of $50 \mu \mathrm{M}$ forskolin causes a rapid increase in the Purkinje cell EPSC. The time course of the peak current is plotted before and after the addition of forskolin (bar). The inset shows averaged EPSCs before (thin line) and after (thick line) exposure to the drug. The time constant of decay of the EPSC in forskolin $(6.1 \mathrm{msec})$ was not significantly changed from that of control $(5.3 \mathrm{msec})$. Traces are the averages of 15-20 trials. $B$, Summary of the mean relative responses $( \pm$ SEM) of synaptic currents to continuous application of $1 \mu \mathrm{M}$ (circles; $207 \pm 38 \% ; n=5$ ), $20 \mu \mathrm{M}$ (squares; $290 \pm 42 \% ; n=10$ ), and $50 \mu \mathrm{M}$ (triangles; $390 \pm 45 \% ; n=11$ ) forskolin and of $50 \mu \mathrm{M}$ 1,9-dideoxyforskolin (diamonds; $115 \pm 15 \% ; n=3$ ). The time course of each experiment is normalized to the average control peak EPSC. Forskolin was added at time 0 .

calcium levels were monitored with fura- 2 using 340 and $380 \mathrm{~nm}$ excitation and the ratio method (Grynkiewicz et al., 1985).

\section{RESULTS}

\section{Forskolin-mediated modulation of evoked and spontaneous synaptic transmission}

Forskolin-mediated changes in synaptic strength were examined at connections between cerebellar granule cells and Purkinje cells. To facilitate voltage control of synaptic responses, we used large electrodes, kept synaptic currents small, and performed experiments on slices from young rats (see Materials and Methods). In all experiments, forskolin was applied continuously to ensure a maximal effect for a given dosage. In Figure $1 A$, the addition of $50 \mu \mathrm{M}$ forskolin resulted in a rapid and sustained enhancement of the peak EPSC from $\sim 150$ to $650 \mathrm{pA}$, without significantly affecting its time course. The enhancement produced by $50 \mu \mathrm{M}$ forskolin was large $(390 \pm 45 \%$ of control; $n=11)$ and variable (198-615\% of control). Figure $1 B$ summarizes the dose dependence of the forskolin-induced enhancement of synaptic 
A

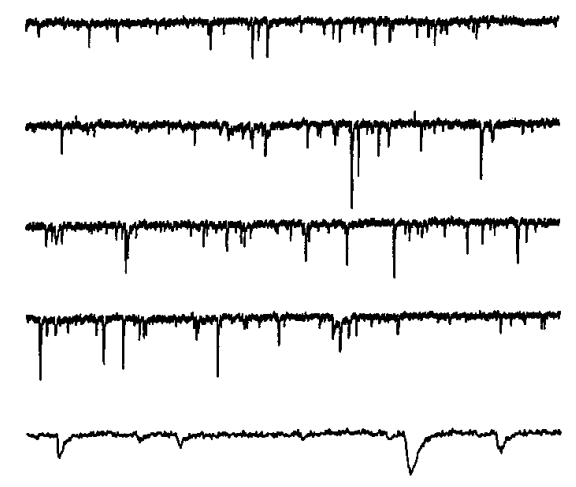

B

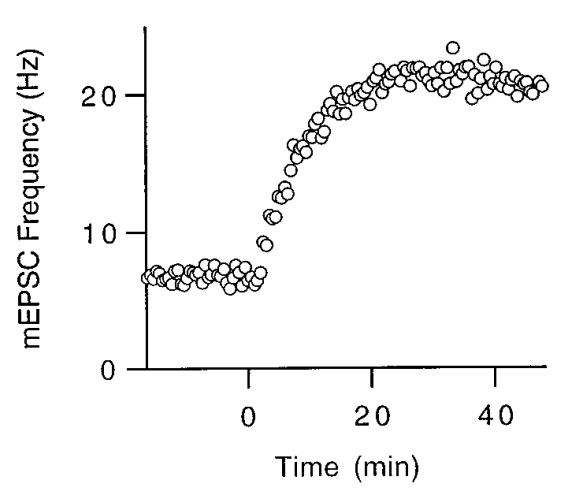

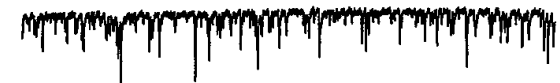

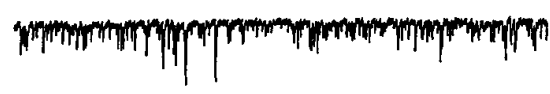

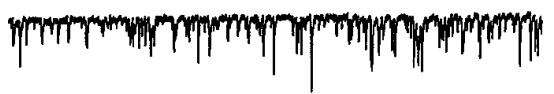
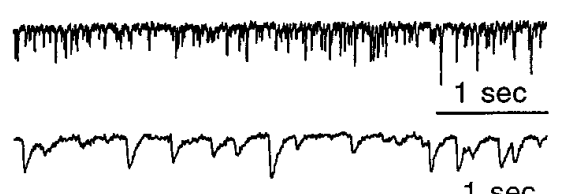

$.1 \mathrm{sec}$

C

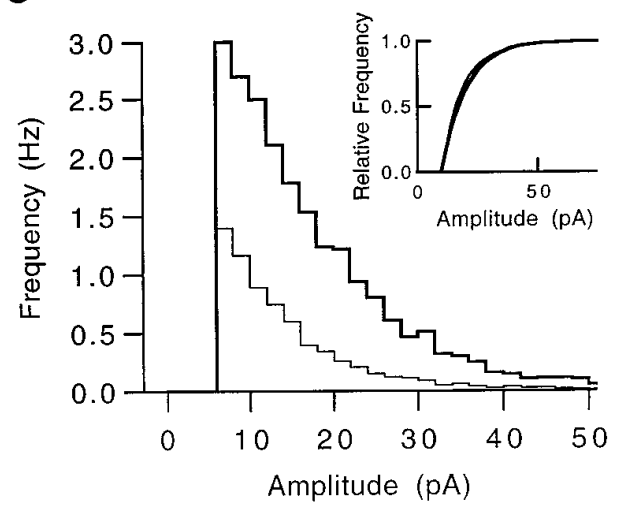

Figure 2. Forskolin enhances spontaneous mEPSCs. $A$, Purkinje cell mEPSCs recorded before (left) and after (right) application of 50 $\mu \mathrm{M}$ forskolin. Unprocessed traces shown in compressed time scale are taken from the last $5 \mathrm{sec}$ of four consecutive $20 \mathrm{sec}$ epochs. Below each side is a trace shown in expanded time scale. $B$, Time course of the average mEPSC frequency in $20 \mathrm{sec}$ epochs during continuous exposure to $50 \mu \mathrm{M}$ forskolin from time 0 . The example is taken from the same experiment shown in $A$. $C$, Amplitude histograms from the same experiment for control (thin line) and $50 \mu \mathrm{M}$ forskolin (bold line) at a holding potential of $-70 \mathrm{mV}$. Inset shows that normalized cumulative amplitude distributions for control (thin line) and forskolin (bold line) are similar $(P=0.85$ by KolmogorovSmirnov test). strength. Synaptic currents began to increase 1-2 min after exposure to forskolin and reached steady state within 7-10 min. In contrast, $50 \mu \mathrm{M}$ 1,9-dideoxy-forskolin, an analog that is ineffective at stimulating adenylate cyclase, did not alter the peak EPSC.

Next we evaluated the effects of forskolin on mEPSCs measured in the presence of TTX. Figure $2 A$ shows a typical response of mEPSCs to $50 \mu \mathrm{M}$ forskolin. Consecutive recordings of mEPSC before (left) and $30 \mathrm{~min}$ after (right) application of forskolin are shown. Bottom traces are shown at an expanded time scale to demonstrate that mEPSCs can be easily resolved. mEPSC frequency increased within a few minutes of forskolin application (Fig. 2B). In this example, the mEPSC frequency increases from 7 to $21 \mathrm{~Hz}$.

The amplitude histograms of mEPSCs for control (thin line) and $50 \mu \mathrm{M}$ forskolin (bold line) are shown in Figure $2 C$. There was a clear increase in the mEPSC frequency at all amplitudes in the presence of forskolin. However, as shown by the normalized cumulative distribution (Fig. $2 C$, inset), there was no significant change in the mEPSC amplitude distribution. To evaluate our ability to detect changes in mEPSC amplitudes, we depolarized the cell at the end of the experiment from -70 to $-50 \mathrm{mV}$, and a statistically significant change in the mEPSC amplitude profile was detected in the normalized amplitude histogram ( $P=0.03$ by Kolmogorov-Smirnov test; data not shown). Thus, in this experiment, forskolin increased mEPSC frequency without affecting mEPSC amplitude.

A summary of the mean relative enhancement of mEPSC frequency at different forskolin doses is shown in Figure 3. In these experiments, the average baseline mEPSC frequency was between 5 and $8 \mathrm{~Hz}$. For 1, 20, and $50 \mu \mathrm{M}$ forskolin, spontaneous
mEPSC frequency increased within 5 min after exposure to the drug and reached steady state in 20-30 min. At all doses of forskolin, amplitude distributions were not significantly different from that of control. In contrast, addition of $50 \mu \mathrm{M} 1,9$-dideoxyforskolin resulted in only a slight increase in mEPSC frequency.

Comparing the actions of forskolin on mEPSC frequency and evoked EPSCs reveals two interesting features; the enhancement of mEPSC reaches steady state more slowly and is less variable than that of evoked EPSCs. Both observations reflect the conditions of these different types of experiments. The evoked EPSCs are produced by activation of a few synapses near the surface of the slice. In contrast, the mEPSCs result from many synapses on all regions of the Purkinje cell, which extends deep within the slice. Consequently, the effects of forskolin reach steady state more slowly in the mEPSC experiments as a result of the time taken for this lipophilic agent to diffuse to sites deep within the slice. Furthermore, the lower variability in the effects of forskolin on mEPSCs likely reflects the contributions of larger numbers of synapses in these experiments compared with the evoked EPSC experiments.

\section{The effects of forskolin on presynaptic calcium influx and fiber excitability}

Because cAMP has been shown to modulate calcium channels and to affect the presynaptic waveform (Hille, 1992), we tested whether increases in presynaptic calcium entry contributed to synaptic enhancement by forskolin. As described previously, low affinity calcium indicators can be used to detect changes in presynaptic calcium entry at the synapse between granule cells and Purkinje cells (Regehr and Atluri, 1995). We labeled parallel 


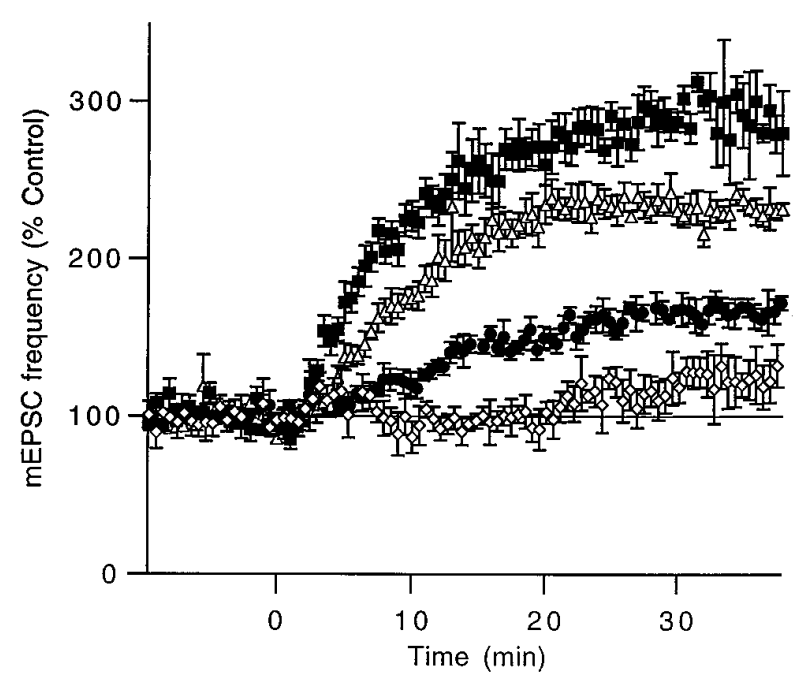

Figure 3. Forskolin enhances mEPSC in a dose-dependent manner. Summary of the average relative responses of spontaneous mEPSCs to application of $1 \mu \mathrm{M}$ (circles; $170 \pm 5 \% ; n=4), 20 \mu \mathrm{M}$ (triangles; $230 \pm 6 \%$; $n=6$ ), and $50 \mu \mathrm{M}$ (squares; $280 \pm 8 \% ; n=4$ ) forskolin as well as $50 \mu \mathrm{M}$ 1,9-dideoxy-forskolin (diamonds; $126 \pm 12 \% ; n=3$ ). Forskolin was applied continuously from time 0 . The time course of each experiment was normalized to the average baseline frequency of control mEPSC. No significant change in the mEPSC amplitude distributions was detected at any dose of forskolin (e.g., at $50 \mu \mathrm{M}$ forskolin, $P=0.88 \pm 0.04 ; n=4$ ).

fibers with the calcium-sensitive indicator magnesium green and assessed the effect of forskolin on the stimulus-evoked changes in fluorescence $(\Delta F / F$ signals). As shown in Figure $4 A, 20 \mu \mathrm{M}$ forskolin increased $\Delta F / F$ signals by $\sim 30 \%$. This enhancement could result from either an elevation of presynaptic calcium entry or a change in fiber excitability. To distinguish between these possibilities, we tested the effect of forskolin on fiber excitability by simultaneously measuring the presynaptic volley (Fig. 4B). The presynaptic volley, which is the field potential associated with the propagating action potential in the parallel fibers, provides a sensitive means of testing for changes in the number of activated fibers (Sabatini and Regehr, 1995, 1997). Forskolin $(20 \mu \mathrm{M})$ increased the amplitude of the presynaptic volley by $\sim 35 \%$, indicating a change in fiber excitability. Forskolin did not significantly affect the waveform of the presynaptic action potential, because the shape of the presynaptic volley was virtually unaltered. However, the latency between stimulus and volley was reduced, suggesting that the conduction velocity was increased (Fig. 4B, inset). As shown in Figure $4 C$, forskolin enhanced the presynaptic volley and $\Delta F / F$ signals with a similar time course and to a comparable degree.

A summary of many such experiments revealed that forskolin had a small effect on both fiber excitability and $\Delta F / F$ signals (Fig. $4 D)$. The dose dependence seemed to differ from that of forskolin-mediated synaptic enhancement, because $20 \mu \mathrm{M}$ forskolin resulted in maximal elevation of fiber excitability. There was an approximately one-to-one correspondence between the changes in fiber excitability and presynaptic calcium (Fig. 4D), suggesting that the small increase in fiber excitability seen in the presence of forskolin accounts for the enhancement seen in $\Delta F / F$ signals.

We reexamined the involvement of changes in presynaptic calcium influx using an approach that is unaffected by changes in fiber excitability (Sabatini and Regehr, 1995). This method capitalizes on the fact that after single action potentials, presynaptic
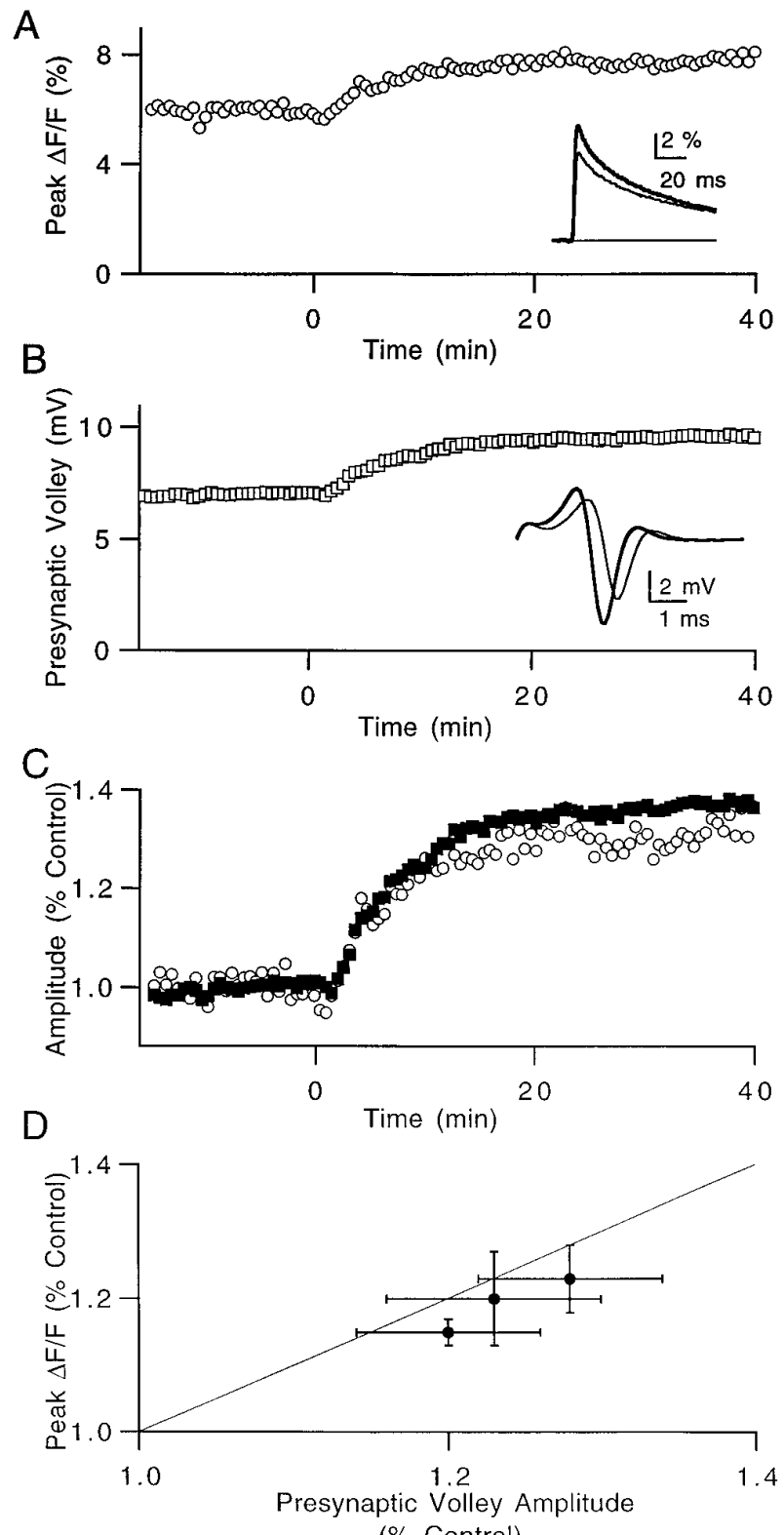

(\% Control)

Figure 4. Effects of forskolin on presynaptic calcium transients. $A, B$, Simultaneous time courses of the amplitude of peak magnesium green fluorescence transients $(A)$ and presynaptic volleys $(B)$ during exposure to $20 \mu \mathrm{M}$ forskolin $(t=0)$. Insets show superimposed average traces of control (thin line) and forskolin (bold line) $\Delta F / F(A)$ and volleys $(B)$. Each trace is an average of 20 trials. The stimulus artifact, determined by the addition of $1 \mu \mathrm{M}$ TTX at the end of the experiment, was subtracted off the averaged traces of presynaptic volleys. $C$, The same time courses shown in $A$ (circles) and $B$ (squares) are normalized to their respective mean control values. Forskolin enhancement of presynaptic volley $(35 \%)$ is very similar to that of presynaptic calcium transients $(30 \%)$. D, Plot of the relative magnesium green fluorescence transient as a function of the relative enhancement of presynaptic volley for (from left to right) $1 \mu \mathrm{M}(n=4), 50$ $\mu \mathrm{M}(n=8)$, and $20 \mu \mathrm{M}(n=4)$ forskolin. Thin line represents $x=y$.

calcium levels are sufficiently large to begin to saturate high affinity calcium indicators, such as fura-2. The degree of saturation is determined by the ratio of the fluorescence changes produced by one and two stimuli $\left[(\Delta F / F)_{2} /(\Delta F / F)_{1}\right]$. This ratio provides a means of quantitating changes in calcium influx that is insensitive to alterations in fiber excitability. An increase in influx 

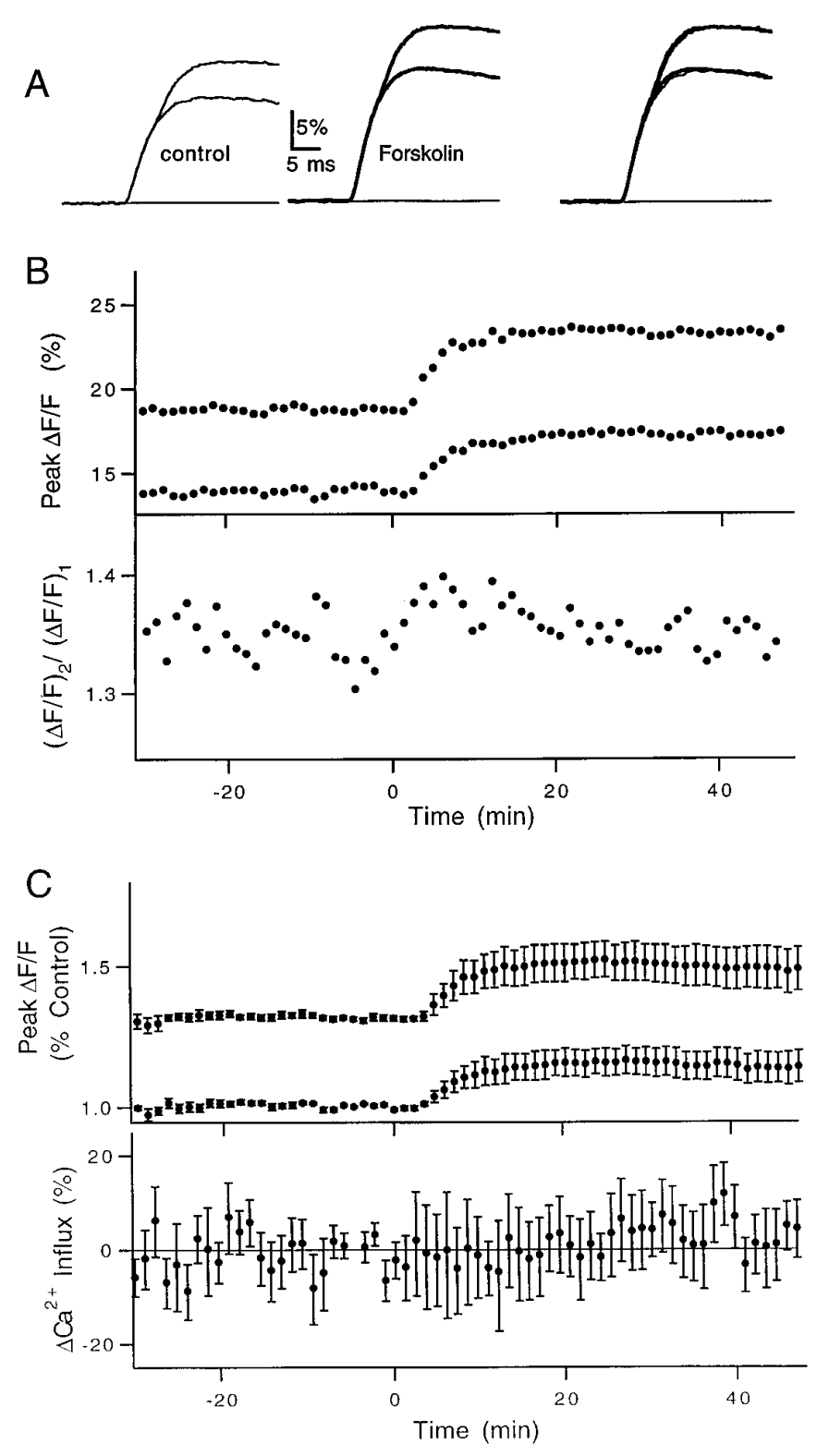

Figure 5. Fura-2 fluorescence measurements of presynaptic calcium influx. A, Average traces of fluorescence changes produced by one stimulus superimposed on that of two stimuli in control conditions (left) and in the presence of $50 \mu \mathrm{M}$ forskolin (middle). Right, The trace for the first stimulus of control is scaled to that of forskolin to emphasize that the relative fluorescence change between one and two stimuli is the same for the two conditions. Traces are the averages of 15-20 trials. $B$, The time course of peak fura- $\Delta, F / F$ for one (lower curve) and two (upper curve) stimuli before and after the addition of forskolin (time 0 ). The relative change in $\Delta F / F$ for two versus one stimuli is plotted below. $C$, Summary of the fura-2 fluorescence changes for four experiments. The effects of forskolin on the relative mean $\Delta F / F$ values for one (lower curve) and two (upper curve) stimuli are plotted as a function of time. The average ratio of $\Delta F / F$ for two versus one stimuli, a direct measurement of the change in calcium influx, is plotted below.

decreases $(\Delta F / F)_{2} /(\Delta F / F)_{1}$. Superimposed average traces of the $\Delta F / F$ transients for one and two stimuli are shown for control conditions in Figure $5 A$ (left). Forskolin increased the amplitude of both $\Delta F / F$ signals (Fig. $5 A$, middle), but the relative size of the two signals was unchanged. This is apparent in Figure $5 A$ (right) in which the traces in control conditions and in forskolin were

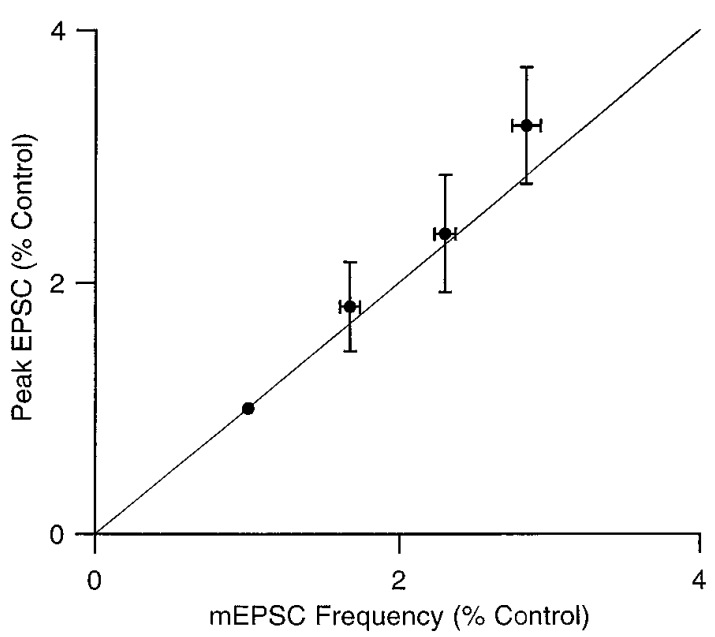

Figure 6. Relationship of forskolin enhancement of evoked to spontaneous release. Plot of the average relative peak EPSC of evoked versus spontaneous responses for (left to right) 0 (theoretical point), 1, 20, and 50 $\mu \mathrm{M}$ forskolin. The relative peak EPSCs were corrected for small enhancements of presynaptic volley by dividing the mean relative evoked enhancement (see Fig. $1 B$ ) by the mean relative $\Delta F / F$ enhancement (see Fig. $4 D$ ) for each forskolin concentration. Thin line represents $x=y$.

normalized to the transients produced by single stimuli. Figure $5 B$ shows the time course of the effect of $50 \mu \mathrm{M}$ forskolin on the fura-2 $\Delta F / F$ changes for one and two stimuli (top). The ratio of $\Delta F / F$ signals produced by one and two stimuli are plotted below on the same time scale. The results of four experiments are summarized in Figure 5C. Presynaptic calcium influx, which is directly determined by the ratio $(\Delta F / F)_{2} /(\Delta F / F)_{1}$, is unaffected by the addition of forskolin. Thus the results of two independent methods show that forskolin does not increase presynaptic calcium influx.

In addition, resting calcium levels were monitored using fura-2 and the ratio method ( $n=4$; data not shown). There was no detectable change in resting ratio, indicating that resting calcium levels were unaltered.

\section{Relationship between evoked and spontaneous EPSCs}

The frequency of spontaneous EPSCs can provide a sensitive means of detecting changes in release that are independent of calcium entry. To examine the contribution of calciumindependent changes to the enhancement of evoked release, we compared the effect of forskolin on evoked currents to its effect on mEPSC frequency. To correct for increases in fiber excitability, we divided evoked currents by the average enhancement of $\Delta F / F$ (magnesium green) for each forskolin dose. As shown in Figure 6, there is a one-to-one correlation between mEPSC frequency and evoked EPSCs at each forskolin dose (from left to right, 0, 1, 20, and $50 \mu \mathrm{M}$ forskolin). This suggests that presynaptic effects independent of calcium influx account for all of the forskolinmediated enhancement of the granule cell to Purkinje cell synapse (see Discussion).

\section{The effect of forskolin on paired-pulse facilitation}

Paired-pulse facilitation (ppf), the short-term enhancement of release that persists for tens of milliseconds after a single conditioning pulse, can be used to detect changes in the probability of release (Zucker, 1973, 1989; Manabe et al., 1993; Atluri and Regehr, 1996). At the granule cell to Purkinje cell synapse, it has been shown previously that in conjunction with an enhancement 
A

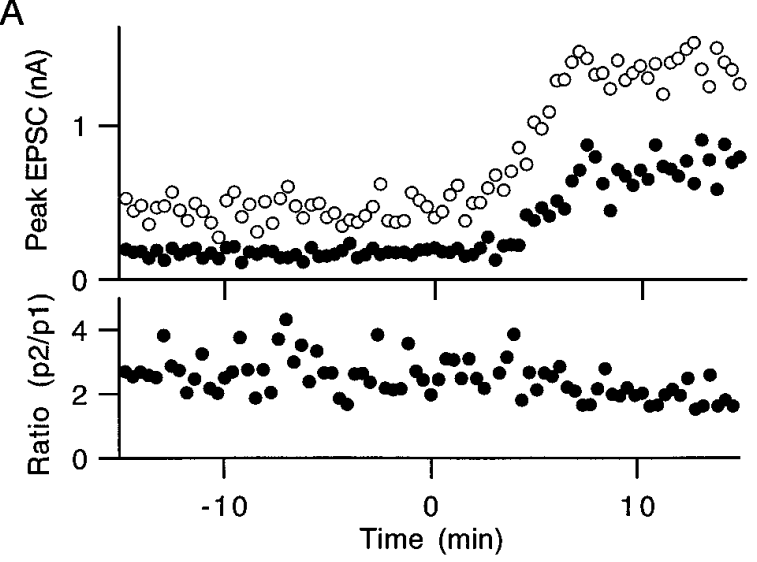

Figure 7. Effects of forskolin on pairedpulse facilitation. $A$, Peak EPSCs of two stimuli separated by $30 \mathrm{msec}$ were plotted as a function of time before and after $50 \mu \mathrm{M}$ forskolin application (time 0 ). The amount of facilitation, recorded as the ratio of the amplitude of the second peak (white) to that of the first peak (black) is shown below. The top right panel shows the average traces for control (thin line) and $50 \mu \mathrm{M}$ forskolin (bold line) currents. Scaling of the first peak of the control trace to the first peak of the forskolin trace shows a decrease of facilitation in the presence of forskolin (bottom right panel). Traces are averages of $10-15$ trials. Stimulus artifacts are blanked for clarity. $B$, Analogous experiment to that described in $A$ except $750 \mu \mathrm{M}$ kynurenic acid is present. $C$, Mean \pm SEM of the relative facilitation before and after the continuous application of $50 \mu \mathrm{M}$ forskolin (circles; $n=4$ ) or 1,9-dideoxy-forskolin (squares; $n=4$ ). Drug application occurred at time 0 . The time course of the facilitated ratio of each experiment was normalized to its mean control value. Each point in the time course of the facilitation ratio represents the average value of three consecutive time points.

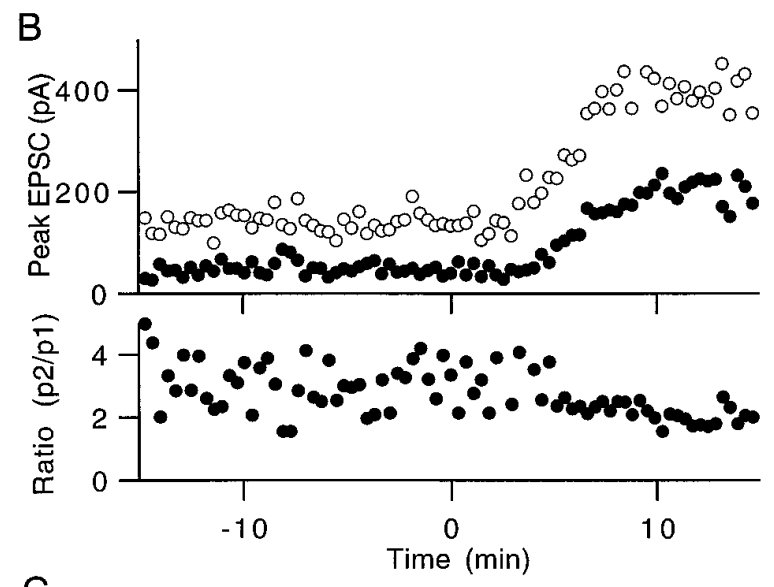

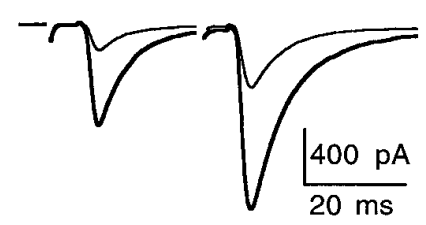
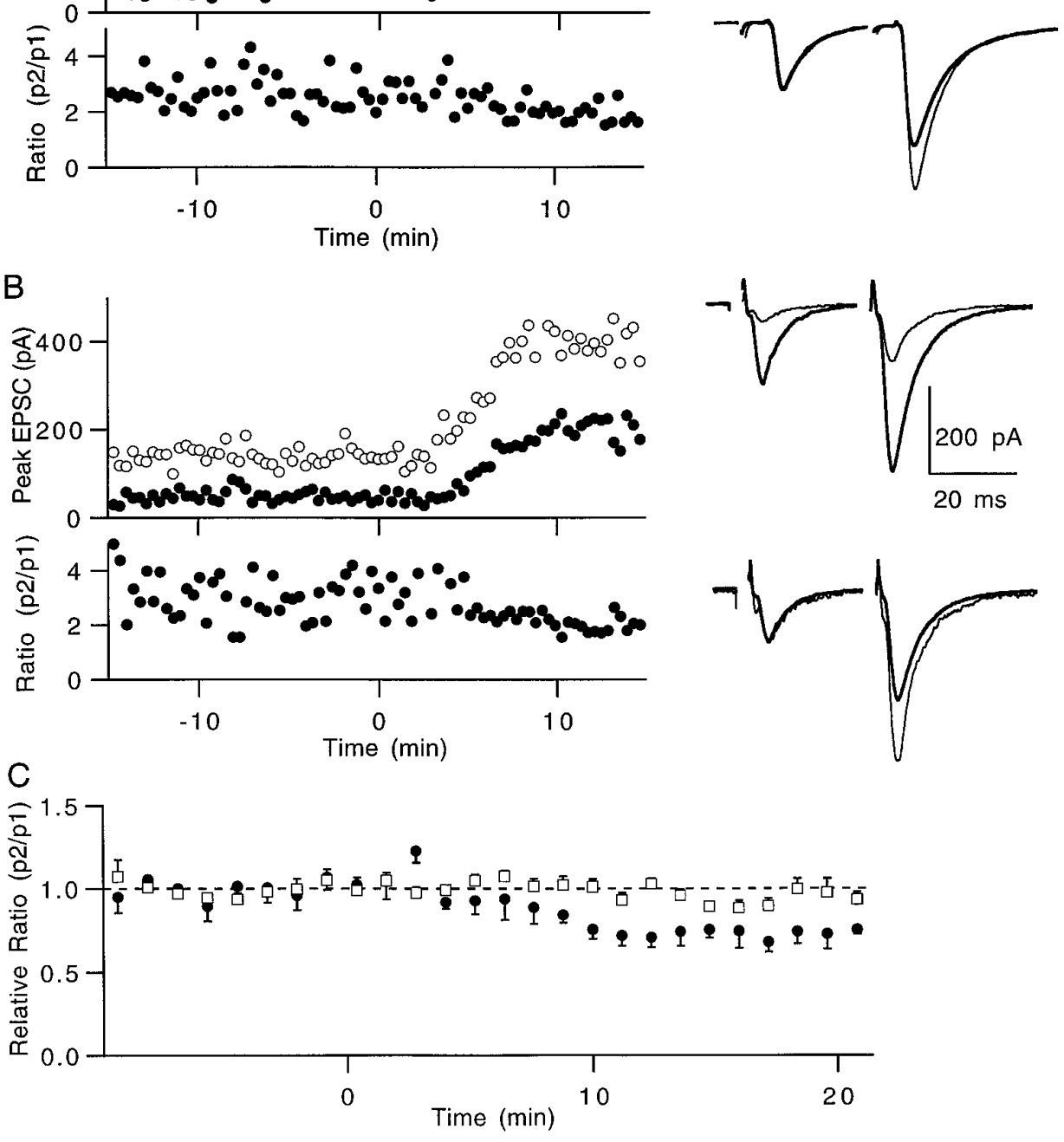

of synaptic strength, a brief application of forskolin decreases ppf (Salin et al., 1996). We examined the effects of forskolin on ppf to determine whether a change in the probability of release $(p)$ was sufficient to account for all of the synaptic enhancement.

We first quantified the effect of a sustained application of forskolin on ppf in $2 \mathrm{~mm}$ external calcium $\left(\mathrm{Ca}_{\mathrm{e}}\right)$. In these experiments, the second synaptic current in forskolin can be more than eightfold larger than the first current in control conditions. This makes accurate quantification of the effect of forskolin on ppf difficult, because large synaptic currents can lead to series resistance errors and an underestimation of ppf, and small synaptic currents are highly variable. One strategy would be to reduce the stimulus strength in the presence of forskolin to minimize the effects of series resistance. However, because different fibers exhibited different degrees of ppf, we believed it was necessary to maintain the same stimulus intensity. We therefore had stringent criteria for the rejection of experiments in which series resistance errors would affect the results. We found that the most sensitive measure of changes in series resistance was the time constant of the decay of the synaptic currents. Experiments were rejected if the time constant of decay of two EPSCs in a pair of pulses differed by $>10 \%$. Figure $7 A$ shows an example of an experiment in which the peak EPSC evoked by each of two stimuli separated by $30 \mathrm{msec}$ was monitored before and after exposure to $50 \mu \mathrm{M}$ forskolin. The facilitation ratio peak2/peak1, which is plotted below on the same time scale, decreased in the presence of forskolin. This decrease in ppf is also apparent when the average EPSCs measured in control conditions and in the presence of forskolin are normalized and superimposed (Fig. 7A, bottom right panel).

We also examined the effects of forskolin on ppf in the presence of KYN $(750 \mu \mathrm{M})$. This allowed us to minimize the synaptic variability and, as shown in Figure $7 B$, to perform experiments with smaller baseline synaptic currents. Neither the degree of facilitation nor the enhancement by $50 \mu \mathrm{M}$ forskolin were altered by KYN. When normalized to the first synaptic current, superposition of the average EPSC in control conditions and in the presence of forskolin shows a clear decrease in ppf in the pres- 
ence of forskolin (Fig. 7B, bottom right panel). A summary of the response of the facilitation ratio to application of $50 \mu \mathrm{M}$ forskolin is shown in Figure $7 C$ (circles). 1,9-Dideoxy-forskolin did not affect ppf (Fig. 7C, squares).

These experiments confirm the finding that forskolin reduces the magnitude of paired-pulse facilitation, indicating that the average $p$ increased. Forskolin $(50 \mu \mathrm{M})$ enhanced evoked EPSCs by $320 \pm 50 \%$ (when corrected for fiber excitability) and decreased ppf by $29 \pm 4 \%(n=4)$. In comparison, increasing $\mathrm{Ca}_{\mathrm{e}}$ from 1 to $2 \mathrm{~mm}$, which increases evoked EPSCs to $330 \%$ by increasing $p$, decreases the facilitation ratio by $37 \%$ (P. P. Atluri and W. G. Regehr, unpublished observations). The similarity in the effect on facilitation for these two manipulations that result in the same degree of enhancement suggests that forskolin acts mainly by increasing $P$.

\section{DISCUSSION}

\section{Calcium-independent presynaptic modulation}

Various approaches were taken to determine the site(s) of action of cAMP in enhancing transmission at the granule cell to Purkinje cell synapse. Based on these experiments, several possible sites of action were ruled out. Resting $\mathrm{Ca}_{\mathrm{i}}$ levels were unchanged. In addition, mEPSC size remained constant, indicating that postsynaptic receptor sensitivity was unaltered. Perhaps most surprisingly, several lines of evidence indicate that changes in presynaptic calcium entry do not contribute to the synaptic enhancement produced by forskolin. There was no significant change in the shape of the presynaptic volley, which is a sensitive means of detecting changes in the presynaptic waveform; this indicates that indirect effects on calcium channel activation resulting from spike broadening do not contribute to cAMPmediated enhancement at this synapse. We also examined the possibility that calcium channel activity was modulated. Although there were small increases in the amplitude of presynaptic $\Delta F / F$ signals for calcium-sensitive indicators, these were a consequence of increases in fiber excitability. Furthermore, an assay that was insensitive to changes in fiber excitability revealed no significant change in calcium influx (an increase of $2.3 \pm 3.7 \%$ ).

Together these experiments established that for our experimental conditions, spike broadening, presynaptic calcium channel modulation, changes in resting calcium levels, and changes in postsynaptic neurotransmitter sensitivity do not contribute to cAMP-dependent plasticity at this synapse. This establishes that forskolin affected synaptic strength exclusively by presynaptic changes downstream from calcium influx. Similar forms of synaptic modulation have been widely reported (Man-Son-Hing et al., 1989; Dale and Kandel, 1990; Delaney et al., 1991; Scanziani et al., 1992); however the molecular sites of action have not been identified. Such synaptic modifications are generally correlated with changes in mEPSC frequency, and this held for forskolin at the granule cell to Purkinje cell synapse.

Although the correlation between altered mEPSC frequency and changes in the amplitude of evoked EPSC supports a contribution from calcium-independent mechanisms to the modulation of many synapses, it has often been difficult to rule out involvement of additional mechanisms. Indeed, in many cases, multiple mechanisms can work together to modify synaptic strength. For example, at this synapse, activation of $\mathrm{GABA}_{\mathrm{B}}$-mediated inhibition is a consequence of decreased presynaptic calcium entry and calcium-independent mechanisms (Dittman and Regehr, 1996). We have shown that the modulation of the granule cell to Purkinje cell synapse by forskolin occurs solely via presynaptic pro- cesses downstream from calcium entry. This study is one of the few examples in which contributions from other mechanisms could be excluded (Delaney et al., 1991; Trudeau et al., 1996).

What is the relationship between mEPSC frequency and the amplitude of evoked EPSCs? Although detection of changes in mEPSC frequency has been a valuable tool in the study of synaptic transmission, the factors governing mEPSC frequency are not fully understood. In many instances, mEPSC frequency and evoked EPSC amplitude are linked, but this is not always the case (Littleton et al., 1993; Broadie et al., 1994; Diantonio and Schwarz, 1994). Only rarely has the relationship between mEPSC frequency and the amplitude of evoked EPSCs been quantitatively examined (Shapira et al., 1987; Abdul-Ghani et al., 1991; Van der Kloot and Molgo, 1994). Here we are able to compare changes in evoked amplitudes and mEPSC frequency produced by forskolin. For these conditions, there is a one-to-one correspondence between the amplitude of evoked EPSCs and mEPSC frequency.

\section{The effect of cAMP on the probability of release and the number of release sites}

Neurotransmitter release depends on the mean probability of release $p$ and on the number of functional release sites $n$. Altering either the number of competent presynaptic or postsynaptic sites will change $n$, as it is defined here. Because paired-pulse facilitation depends on the initial probability of release, it provides a means of distinguishing between changes in $p$ and $n$. An increase in $p$ will decrease ppf, whereas an increase in $n$ does not affect ppf. The decrease in ppf observed in $2 \mathrm{~mm} \mathrm{Ca}_{\mathrm{e}}$ indicates that an increase in $p$ contributes to the synaptic enhancement produced by forskolin, consistent with previous reports at this synapse (Salin et al., 1996). Furthermore, the similarities between the effects of forskolin and changing $\mathrm{Ca}_{\mathrm{e}}$ on ppf also suggest that forskolin acts primarily by increasing $p$.

cAMP has been shown to modulate other synapses by increasing both $p$ and $n$. At the crayfish neuromuscular junction and dentate granule cell synapses, tetanic stimulation produces sustained enhancement of synaptic strength that is mediated by cAMP and PKA. In crayfish, increases in $p$ account for $\sim 80 \%$ of this enhancement, whereas an increase in the mean number of active release sites (presumably corresponding to $n$ ) accounts for the rest (Wojtowicz et al., 1988). cAMP also regulates release from dentate granule cells in the hippocampus by changing both $n$ and $p$ (Weisskopf et al., 1994; Tong et al., 1996). In our experiments, it seems that enhancement is primarily a consequence of increases in $p$, although it is not possible to rule out a small contribution from changes in $n$ as well.

In summary, cAMP acts presynaptically, at a site downstream from calcium entry, to enhance transmission at the granule cell to Purkinje cell synapse. Most of this enhancement is a result of increases in $p$.

\section{REFERENCES}

Abdul-Ghani M, Kravitz EA, Meiri H, Rahamimoff R (1991) Protein phosphatase inhibitor okadaic acid enhances transmitter release at neuromuscular junctions. Proc Natl Acad Sci USA 88:1803-1807.

Atluri PP, Regehr WG (1996) Determinants of the time course of facilitation at the granule cell to Purkinje cell synapse. J Neurosci 16:5661-5671.

Broadie K, Bellen HJ, DiAntonio A, Littleton JT, Schwarz TL (1994) Absence of synaptotagmin disrupts excitation-secretion coupling during synaptic transmission. Proc Natl Acad Sci USA 91:10727-10731.

Byrne JH, Kandel ER (1996) Presynaptic facilitation revisited: state and time dependence. J Neurosci 16:425-435. 
Capogna M, Gahwiler BH, Thompson SM (1995) Presynaptic enhancement of inhibitory synaptic transmission by protein kinases $\mathrm{A}$ and $\mathrm{C}$ in the rat hippocampus in vitro. J Neurosci 15:1249-1260.

Chavez-Noriega LE, Stevens CF (1994) Increased transmitter release at excitatory synapses produced by direct activation of adenylate cylase in rat hippocampal slices. J Neurosci 14:310-317.

Dale N, Kandel ER (1990) Facilitatory and inhibitory transmitters modulate spontaneous transmitter release at cultured Aplysia sensorimotor synapses. J Physiol (Lond) 421:203-222.

Delaney K, Tank DW, Zucker RS (1991) Presynaptic calcium and serotonin-mediated enhancement of transmitter release at crayfish neuromuscular junction. J Neurosci 11:2631-2643.

Diantonio A, Schwarz TL (1994) The effect on synaptic physiology of synaptotagmin mutations in Drosophila. Neuron 12:909-920.

Dittman JS, Regehr WG (1996) Contributions of calcium dependent and calcium independent mechanisms to presynaptic inhibition at a cerebellar synapse. J Neurosci 16:1623-1633.

Dixon D, Atwood HL (1989) Adenylate cyclase system is essential for long-term facilitation at the crayfish neuromuscular junction. J Neurosci 9:4246-4252.

Goy M, Kravitz EA (1989) Cyclic AMP only partially mediates the actions of serotonin at the lobster neuromuscular junctions. J Neurosci 9:369-379.

Greengard P, Jen J, Nairn AC, Stevens CF (1991) Enhancement of the glutamate response by cAMP-dependent protein kinase in hippocampal neurons. Science 253:1135-1138.

Grynkiewicz G, Poenie M, Tsien RY (1985) A new generation of $\mathrm{Ca}^{2+}$ indicators with greatly improved fluorescence properties. J Biol Chem 260:3440-3450.

Hille B (1992) Ionic channels of excitable membranes, Ed 2. Sunderland, MA: Sinauer.

Huang Y-Y, Li X-C, Kandel E (1994) cAMP contributes to mossy fiber LTP by initiating both a covalently mediated early phase and macromolecular synthesis-dependent late phase. Cell 79:69-79.

Kondo S, Marty A (1997) Protein kinase A-mediated enhancement of miniature IPSC frequency by noradrenaline in rat cerebellar stellate cells. J Physiol (Lond) 498:165-176.

Littleton JT, Stern M, Schulze K, Perin M, Bellen HJ (1993) Mutational analysis of Drosophila synaptotagmin demonstrates its essential role in $\mathrm{Ca}^{2+}$-activated neurotransmitter release. Cell 74:1125-1134.

Llano I, Gerschenfeld H (1993) $\beta$-Adrenergic enhancement of inhibitory synaptic activity in rat cerebellar stellate and Purkinje cells. J Physiol (Lond) 468:201-224.

Llano I, Marty A, Armstrong CM, Konnerth A (1991) Synaptic- and agonist-induced excitatory currents of Purkinje cells in rat cerebellar slices. J Physiol (Lond) 434:183-213.

Man-Son-Hing H, Zoran MJ, Lukowiak K, Haydon PG (1989) A neuromodulator of synaptic transmission acts on the secretory apparatus as well as on ion channels. Nature 341:237-239.

Manabe T, Wyllie DJ, Perkel DJ, Nicoll RA (1993) Modulation of synaptic transmission and long-term potentiation: effects on paired pulse facilitation and EPSC variance in the CA1 region of the hippocampus. J Neurophysiol 70:1451-1459.
Mintz IM, Sabatini BL, Regehr WG (1995) Calcium control of transmitter release at a cerebellar synapse. Neuron 15:675-688.

Regehr WG, Atluri PP (1995) Calcium transients in cerebellar granule cell presynaptic terminals. Biophys J 68:2156-2170.

Regehr WG, Tank DW (1991) Selective fura-2 loading of presynaptic terminals and nerve cell processes by local perfusion in mammalian brain slice. J Neurosci Methods 37:111-119.

Sabatini BL, Regehr WG (1995) Detecting changes in calcium influx which contribute to synaptic modulation in mammalian brain slice. Neuropharmacology 34:1453-1467.

Sabatini BL, Regehr WG (1997) Control of neurotransmitter release by presynaptic waveform at the granule cell to Purkinje cell synapse. J Neurosci 17:3425-3435.

Salin PA, Malenka RC, Nicoll RA (1996) Cyclic AMP mediates a presynaptic form of LTP at cerebellar parallel fiber synapses. Neuron 16:797-803.

Scanziani M, Copogna M, Gahwiler BH, Thompson SM (1992) Presynaptic inhibition of miniature excitatory synaptic currents by baclofen and adenosine in the hippocampus. Neuron 9:919-927.

Shapira R, Silberberg SD, Ginsburg S, Rahamimoff R (1987) Activation of protein kinase $\mathrm{C}$ augments evoked transmitter release. Nature 325:58-60.

Tong G, Malenka RC, Nicoll R (1996) Long-term potentiation in cultures of single hippocampal granule cells: a presynaptic form of plasticity. Neuron 16:1147-1157.

Trudeau L-E, Emery DG, Haydon PG (1996) Direct modulation of the secretory machinery underlies PKA-dependent synaptic facilitation in hippocampal neurons. Neuron 17:789-797.

Van der Kloot W, Molgo J (1994) Quantal acetylcholine release at the vertebrate neuromuscular junction. Physiol Rev 74:899-991.

Weisskopf MG, Nicoll RA (1995) Presynaptic changes during mossy fiber LTP revealed by NMDA receptor-mediated synaptic responses. Nature 376:256-259.

Weisskopf MG, Castillo PE, Zalutsky RA, Nicoll RA (1994) Mediation of hippocampal mossy fiber long-term potentiation by cyclic AMP. Science 265:1878-1882.

Wojtowicz JM, Parnas I, Parnas H, Atwood HL (1988) Long-term facilitation of synaptic transmission demonstrated with macro-patch recording at the crayfish neuromuscular junction. Neurosci Lett 90:152-158.

Wojtowicz JM, Marin L, Atwood HL (1994) Activity-induced changes in synaptic release sites at the crayfish neuromuscular junction. J Neurosci 14:3688-3703.

Zhang G, Lui Y, Ruoho A, Hurley J (1997) Structure of the adenylyl cyclase catalytic core. Nature 386:247-253.

Zhao M, Hollingworth S, Baylor SM (1996) Properties of tri- and tetracarboxylate $\mathrm{Ca}^{2+}$ indicators in frog skeletal muscle fibers. Biophys $\mathbf{J}$ 70:896-916.

Zucker R (1973) Changes in the statistics of transmitter release during facilitation. J Physiol (Lond) 229:787-810.

Zucker RS (1989) Short-term synaptic plasticity. Annu Rev Neurosci 12:13-31. 